\title{
Surface-enhanced vibrational spectroscopy for probing transient interactions of proteins with biomimetic interfaces: electric field effects on structure, dynamics and function of cytochrome $c$
}

\author{
Hong Khoa Ly ${ }^{1}$, Murat Sezer ${ }^{1}$, Nattawadee Wisitruangsakul ${ }^{1,2}$, Jiu-Ju Feng ${ }^{1,3}$, Anja Kranich ${ }^{1}$, \\ Diego Millo ${ }^{1}$, Inez M. Weidinger ${ }^{1}$, Ingo Zebger ${ }^{1}$, Daniel H. Murgida ${ }^{4}$ and Peter Hildebrandt ${ }^{1}$ \\ 1 Technische Universität Berlin, Institut für Chemie, Germany \\ 2 Iron and Steel Institute of Thailand, Bangkok, Thailand \\ 3 School of Chemistry and Environmental Science, Henan Normal University, Xinxiang, China \\ 4 Departamento de Química Inorgánica, Analítica y Química Física/INQUIMAE-CONICET, Facultad de Ciencias Exactas y Naturales, Universi- \\ dad de Buenos Aires, Argentina
}

\author{
Keywords \\ apoptosis; cytochrome c; electric field; \\ electron transfer; protein dynamics; surface- \\ enhanced infrared spectroscopy; surface- \\ enhanced resonance Raman spectroscopy

\section{Correspondence \\ P. Hildebrandt, Technische Universität Berlin, Institut für Chemie, Sekr. PC 14, Straße des 17 Juni 135, D-10623 Berlin, Germany \\ Fax: +493031421122 \\ Tel: +493031421419 \\ E-mail: Hildebrandt@chem.tu-berlin.de}

(Received 23 November 2010, revised 21 January 2011, accepted 22 February 2011)

doi:10.1111/j.1742-4658.2011.08064.x
Most of the biochemical and biophysical processes of proteins take place at membranes, and are thus under the influence of strong local electric fields, which are likely to affect the structure as well as the reaction mechanism and dynamics. To analyse such electric field effects, biomimetic interfaces may be employed that consist of membrane models deposited on nanostructured metal electrodes. For such devices, surface-enhanced resonance Raman and IR absorption spectroscopy are powerful techniques to disentangle the complex interfacial processes of proteins in terms of rotational diffusion, electron transfer, and protein and cofactor structural changes. The present article reviews the results obtained for the haem protein cytochrome $c$, which is widely used as a model protein for studying the various reaction steps of interfacial redox processes in general. In addition, it is shown that electric field effects may be functional for the natural redox processes of cytochrome $c$ in the respiratory chain, as well as for the switch from the redox to the peroxidase function, one of the key events preceding apoptosis.

\section{Introduction}

Transient interactions of proteins with reaction partners play a key role in biochemical and biophysical processes [1-3]. They govern the formation of encounter complexes between proteins, preceding, for instance, interprotein electron transfer reactions. These interactions may include different elementary steps, such as lateral diffusion of the reactant along the surface of a macromolecular target, a reorientation corresponding to a rotational diffusion within the interaction domain, and eventually mutual conformational changes opening favourable reaction pathways for the subsequent processes. Additional constraints exist for transient interactions at membranes, where most of the biological processes take place. Here, high

\section{Abbreviations}

RR, resonance Raman; SAM, self-assembled monolayer; SEIRA, surface-enhanced infrared absorption; SERR, surface-enhanced resonance Raman; TR, time-resolved. 
local electric fields constitute reaction conditions that differ substantially from those in the solution phase [4]. Specifically, in the interfacial region between the hydrophobic core of the lipid bilayer and the polar or charged head groups, large changes of the potential over a short distance lead to local electric fields as high as $10^{9} \mathrm{~V} \cdot \mathrm{m}^{-1}$ [5], which are expected to have a strong impact on proteins transiently bound at membrane interfaces or to integral membrane proteins. Such high electric fields may perturb acid-base equilibrium, and induce and align molecular dipoles in the macromolecule [6], thereby causing structural changes within the macromolecule that may eventually affect the reaction mechanism and dynamics. This may be particularly true for processes involving the translocation of charges, such as proton or electron transfer.

It is not surprising that our understanding of the biomolecular processes under the influence of electric fields is in its infancy, as dedicated experimental techniques are required. This is particularly true for processes at membranes, as high demands are imposed on the sensitivity and selectivity of the methodology, which should provide molecular structure and dynamics information.

In this article, we present the potential of Raman and IR spectroscopic techniques for probing surfaceconfined processes in membrane models that are designed to mimic important properties of biological interfaces. The first part of this minireview is thus dedicated to the methodology and the concept for studying electric field effects of immobilized proteins. The second part summarizes the results that have been obtained for the mammalian (horse heart) haem protein cytochrome $c$. Cytochrome $c$ is a soluble protein exerting its functions at the interface of the inner mitochondrial membrane. It primarily acts as an electron carrier, delivering electrons from complex III to complex IV, which are both integral membrane enzyme complexes [7]. In addition, cytochrome $c$ has been shown to play a crucial role in apoptotic processes [8], presumably initiated by a structural transition of the protein that abolishes its redox function and strongly increases peroxidase activity [9]. In the third part, we will discuss the impact of the present biomimetic approach on understanding the physiological processes of cytochrome $c$.

\section{Methodology}

Raman and IR spectroscopy are molecular structuresensitive techniques, as the frequencies and band intensities of vibrational transitions represent a unique fingerprint of the specific conformation of a molecule
[10]. Both techniques, however, are associated with low sensitivity and selectivity. For Raman spectroscopy, this drawback can be overcome by choosing excitation lines in resonance with an electronic transition of the cofactor of the protein, to selectively enhance those Raman bands that originate from the chromophoric part of the macromolecule [resonance Raman (RR)]. IR spectroscopy is preferentially employed in the difference mode, such that only those IR bands are monitored that are different between two protein states. In this way, both the sensitivity and the selectivity are substantially increased, as IR difference and RR spectroscopy solely probe the vibrational modes reflecting a reaction of the protein and originating from its active site, respectively. An additional increase in sensitivity, required to probe proteins bound to or integrated in membrane models, is achieved by exploiting the enhancement of optical processes via coupling of the radiation field with surface plasmons of nanostructured Ag or Au. These surface-enhanced RR (SERR) and surface-enhanced IR absorption (SEIRA) difference spectroscopies allow probing molecules even at submonolayer coverages of surfaces [10].

A particular advantage of SERR and SEIRA spectroscopy is that the signal-amplifying support material may also be used as a working electrode when integrated in an electrochemical cell [10-12]. Then, the metal support may serve as an electron supply or sink for electron transfer reactions to or from an immobilized redox protein. In addition, variation of the electrode potential is also one parameter that can alter the local electric field strength experienced by the bound proteins. For such spectroelectrochemical applications, $\mathrm{Au}$ would be the metal of choice, as the applicable potential range is distinctly wider than for $\mathrm{Ag}$. In fact, the optical properties, which control the surface enhancement, are very good in the IR region, such that SEIRA experiments are usually carried out on thin Au films [10,12]. However, on the short-wavelength side of the spectrum, optical excitation of surface plasmons of $\mathrm{Au}$ is restricted to the region above $\sim 550 \mathrm{~nm}$ [10]. This limitation has severe consequences for SERR spectroscopy: since the electronic transitions of most of the protein cofactors (e.g. haem) of proteins are at shorter wavelengths, the combination of molecular RR and the surface-enhanced Raman effect that provides the unique selectivity and sensitivity for the cofactor of the immobilized proteins is only possible with nanostructured $\mathrm{Ag}$ for direct excitation of surface plasmons down to $400 \mathrm{~nm}$. With the recent discovery of the coupling of surface plasmons in layered systems, however, it is now possible to use Ag solely as a signal amplifier while the redox protein interacts with a 
different material such as $\mathrm{Au}$, which also serves as the (primary) electrochemical reaction partner [13-15]. These layered hybrid electrodes are based on a nanostructured $\mathrm{Ag}$ support, covered with a dielectric thin film $(2-20 \mathrm{~nm})$ made of a self-assembled monolayer (SAM) of mercaptans or of silica. This film is then coated by an $\mathrm{Au}$ layer (circa $20 \mathrm{~nm}$ in thickness). Recent studies have demonstrated, that in such devices, the RR signal amplification of the redox proteins immobilized on the outer Au film is only slightly lower than that determined for adsorption on the $\mathrm{Ag}$ support [14].

However, regardless of the type of metal, the direct binding of proteins on solid supports bears the risk of irreversible denaturation. Biocompatible coatings on the metal surface can avoid these unwanted side reactions $[4,16]$. Although such coatings increase the distance of the redox protein from the metal surface, the attenuation of the spectroscopic signals does not usually impair the measurement of SERR and SEIRA spectra with high signal-to-noise ratios.

\section{Concept}

Particularly interesting biocompatible coating materials for metal electrodes are monolayers and bilayers of lipid analogues, as they allow mimicking biological surfaces appropriate for protein binding [16]. For soluble proteins, SAMs of $\omega$-functionalized alkanethiols or disulphides represent the most versatile immobilization platform [17]. These amphiphiles can form densely packed layers, specifically on Au or Ag. SAMs carrying an excess of negative or positive charges can be created by using protonable tail groups, and are particularly suited for electrostatic immobilization of soluble proteins. In the case of the highly cationic cytochrome $c$, SAMs containing carboxyl-terminated mercaptans are preferentially employed [16]. In this sense, SAM-coated electrodes are able to mimic some basic features of biological interfaces as far as Coulombic interactions are concerned. Specifically, the interfacial potential distribution is likely to be very similar for the electrode-SAM and the bilayer systems (Fig. 1) $[4,5]$. In both cases, the region between the hydrophobic core and the polar/charged head groups is characterized by a steep potential gradient corresponding to high local electric fields. Furthermore, the local electric field strength in the electrochemical systems can readily be controlled by changing various parameters. An increase in the electric field strength at the SAM-solution interface, i.e. at the position of protein binding, is achieved by: (a) increasing the difference between the electrode potential and the potential



Fig. 1. Schematic presentation of the potential distribution at the membrane-solution interface (top) and at the electrode-SAM interface (bottom). $\psi_{S}, \psi_{D}$ and $\Delta \psi$ denote the surface potential, dipole potential and transmembrane potential, respectively; $\Delta \phi$ is the difference between electrode potential $(E)$ and the potential of zero charge $\left(E_{\mathrm{pzc}}\right)$.

of zero charge; (b) decreasing the SAM thickness; and (c) increasing the charge density on the SAM surface $[4,17]$.

For a comprehensive analysis of electric field effects, a quantification of the field strength is highly desirable. The direct experimental determination of local electric field strengths is possible on the basis of the vibrational Stark effect $[18,19]$. A particularly appropriate vibrational Stark effect probe is the nitrile function, as the electric field response of the respective stretching frequency and its sensitivity towards environmental factors such as hydrogen bond interactions are well understood [20,21]. SAMs containing nitrile-terminated mercaptans may be used for coating $\mathrm{Ag}$ and $\mathrm{Au}$, such that the nitrile stretching can be monitored by surfaceenhanced vibrational spectroscopy [22]. Preliminary results obtained for mixed SAMs of nitrile-terminated and carboxyl-terminated mercaptans have afforded local electric field strengths that are comparable to those estimated on the basis of simple electrostatic calculations [23]. In addition, the nitrile function may be attached to cysteines of the protein introduced at 
selected positions by site-directed mutagenesis [19]. IR and SEIRA spectroscopy then allow probing the local electric field strength of the protein in solution and in the immobilized state, respectively. In this way, it is possible to map the electric field strength across the electrode-SAM-protein interface, which is a prerequisite for a comprehensive quantitative description of electric field effects on protein structure, dynamics, and function.

\section{Information provided by surface- enhanced vibrational spectroscopy}

Redox proteins immobilized on SAM-coated Ag and $\mathrm{Au}$ electrodes are usually studied by cyclic voltammetry [24]. This technique monitors the current flow as a function of the electrode potential, and thus probes the processes of redox-active proteins. However, it does not provide information about the molecular mechanism of the interfacial processes, which, on the other hand, is accessible with the structure-sensitive SERR and SEIRA spectroscopy. To probe the dynamics of molecular structure changes during the redox process, these techniques may be coupled with the potential jump technique [25]. In this approach, a rapid potential jump is applied to the working electrode, leading to a perturbation of the equilibrium at the initial potential. The subsequent relaxation processes that restore thermodynamic equilibrium at the final potential may then be probed by SERR and SEIRA spectroscopy, the latter being operated in the step scan or rapid scan mode for probing processes faster or slower than $\sim 100 \mathrm{~ms}$, respectively [26].

SERR and SEIRA spectroscopic techniques provide different kinds of information about the interfacial processes of cytochrome $c$. First, the unique vibrational signatures of reduced and oxidized haems allows the two oxidation states of the immobilized cytochrome $c$ to be distinguished. The respective marker bands mainly originate from totally symmetric modes that are selectively enhanced when the excitation line is in resonance with the strongly allowed Soret transition $[10,23,25]$. Thus, SERR spectra obtained with 413-nm excitation allow for the determination of the redox equilibria and, in the time-resolved (TR) mode, the electron transfer kinetics of the immobilized cytochrome $c$ (Fig. 2).

Second, the frequencies of these marker bands not only respond to changes in the oxidation state of the central haem iron, but also reflect alterations in the coordination sphere, such that these marker bands allow monitoring equilibria and dynamics of the spin, coordination and ligation configuration of cytochrome $c[10,27]$.

Third, when excitation lines close to the weak Q-transition of the haem are used, the surface enhancement of totally symmetric $\left(\mathrm{A}_{1 \mathrm{~g}}\right)$ and nontotally symmetric (e.g. $\mathrm{B}_{1 \mathrm{~g}}$ ) modes of the haem depends on its orientation relative to the surface [28]. As the haem is fixed in the protein matrix, the relative intensities of $\mathrm{B}_{1 \mathrm{~g}}$ and $\mathrm{A}_{1 \mathrm{~g}}$ modes in these Q-bandexcited SERR spectra may be used as a spectral
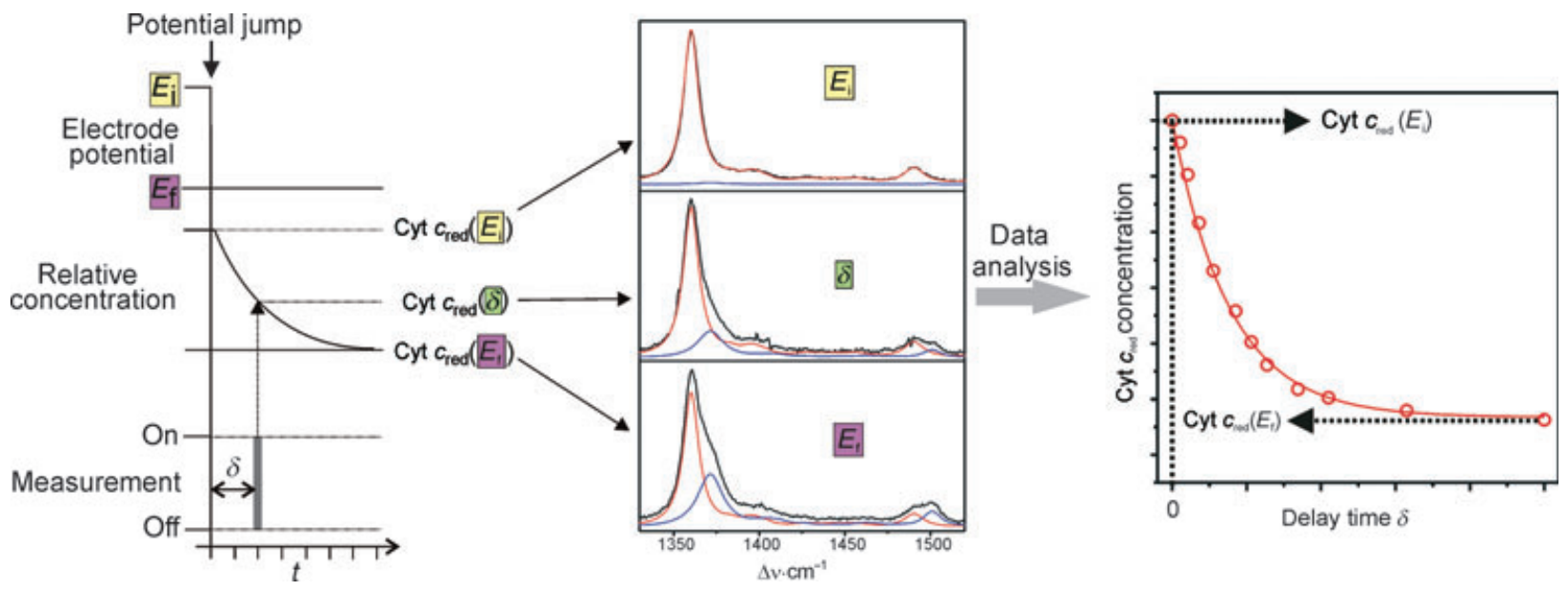

Fig. 2. Schematic presentation of the potential jump time-resolved SERR experiment for a potential jump from negative to positive potentials. Left: temporal relationship between potential jump, concentration changes, and measurements. Middle: SERR spectra measured at the initial potential $E_{\mathrm{i}}$ (top), the final potential $E_{\mathrm{f}}$ (bottom), and after a delay time $\delta$ following the potential jump from $E_{\mathrm{i}}$ to $E_{\mathrm{f}}$; the red and blue lines refer to the component spectra of the reduced and oxidized cytochrome $c$ (Cyt $c)$, respectively. Right: results of the spectral analysis showing the relaxation of the reduced cytochrome $c$ following the potential jump. Further details are given in [25]. 
marker for tracing changes in the average orientation of the immobilized protein.

Fourth, SEIRA spectroscopy provides complementary information about redox-linked structural changes of the protein and orientation changes of individual peptide segments [12,26]. SEIRA experiments are carried out in the difference mode. The spectra measured at various electrode potentials are related to a reference spectrum obtained at a fixed potential, such that the difference spectra display only those bands that undergo potential-dependent changes. The characteristic marker bands for this technique are the amide I modes, the frequencies of which are indicative for the various secondary structure elements of cytochrome $c$.

\section{Dynamics of the interfacial redox process}

For 20 years, SAM-coated electrodes have been used as a convenient platform for studying biological electron transfer reactions [17]. Special attention has been paid to the analysis of the distance dependence of the heterogeneous electron transfer upon variation of the SAM thickness, using cytochrome $c$ as a model protein $[25,26,28-37]$. It was found that, with decreasing distance, the electron transfer rate constant first increases exponentially, as expected for long-range electron tunnelling, but then levels off to reach a plateau. Qualitatively, the same findings were obtained with both electrochemical methods such as cyclic voltammetry, which probe the electron flow between the immobilized cytochrome $c$ and the electrode, and SERR spectroscopy with Soret band excitation, which monitors the change in the oxidation state of the haem (Fig. 3). However, by means of the various surface-enhanced vibrational spectroscopic approaches, it is possible to monitor further elementary reaction steps of the immobilized protein that are coupled to electron tunnelling. TR-SEIRA spectroscopy reveals that small protein structural changes occur concomitantly with electron transfer [26]. These changes are reflected by bands that have also been detected in redox-induced IR difference spectra of cytochrome $c$ in solution. The most prominent spectral changes have been attributed to the $\beta$-turn III peptide segment 67-70 [26]. However, no structural changes that might account for the unique kinetic behaviour are detectable by SERR or SEIRA spectroscopy. On the other hand, protein orientation changes show a distance dependence that deviates from the electron tunnelling kinetics [28]. At SAMs of mercaptohexadecanoic acid including 15 methylene groups in the alkyl chain (C15-SAM), potential jump-induced protein reorientation, as probed by TR-SERR

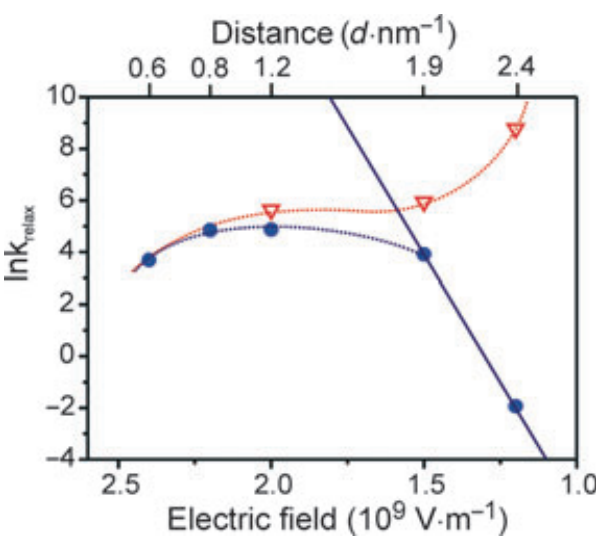

Fig. 3. Rate constants for reorientation (red) and reduction (blue) of cytochrome $c$ immobilised on Ag electrodes coated with carboxylterminated SAMs of different chain lengths, determined by timeresolved SERR spectroscopy $[25,28,40]$. The bottom axis indicates the electric field strength at the SAM-cytochrome $c$ interface as estimated on the basis of an electrostatic model [23]. The straight line represents the exponential distance dependence of electron tunnelling, extrapolated from rate constants determined for C15SAM and C10-SAM. The dotted lines are included to guide the eyes.

experiments with Q-band excitation, is much faster than electron tunnelling (Fig. 3). With a decreasing number of methylene groups, the rate of protein reorientation decreases until it approaches the rate of electron tunnelling. At SAMs of mercaptohexanoic acid (C5-SAM), the rate constants determined for orientation changes and electron tunnelling are essentially the same.

Accordingly, one may distinguish two different regimes for the interfacial electron transfer of the immobilized cytochrome $c$ : at long distances, at SAMs with 10 or more methylene groups, electron transfer is solely controlled by electron tunnelling, whereas at shorter distances, orientation changes of the immobilized protein appear to be rate-limiting. In view of the distance dependence of the interfacial electric field, one may alternatively classify the redox process in terms of a low-field (long distances) and a high-field (short distances) regime.

\section{Electron transfer in the low-field regime}

Despite the low number of experimental data points in the low-field regime, both in electrochemical and in spectroelectrochemical measurements, one may conclude that the kinetic data follow the expected exponential distance dependence for electron tunnelling. Furthermore, overpotential-dependent and 
temperature-dependent measurements for cytochrome $c$ immobilized on C15-SAM-coated Ag electrode (TRSERR) are consistent with the Marcus theory for long-range electron tunnelling [38]. The reorganization energy derived from these studies is distinctly lower than that determined for cytochrome $c$ in solution [39], indicating a strongly reduced contribution of the solvent reorganization in the immobilized state [38]. However, much weaker overpotential dependencies, corresponding to physically meaningless low values for the reorganization energy, are obtained for SAMcoated $\mathrm{Au}$ and $\mathrm{Au}-\mathrm{Ag}$ hybrid instead of $\mathrm{Ag}$ electrodes [40]. In an attempt to reconcile these conflicting results, it has been proposed that, also in the low-field regime, the local electric field at the SAM-cytochrome $c$ interface affects the electron transfer step. Assuming that the local electric field strength is proportional to the difference between the actual electrode potential and the metal-specific potential of zero charge, an empirical linear correction has been included in the free-energy term of the Marcus equation, in analogy to previous approaches employed for describing field effects on intramolecular electron transfer reactions [41]. This rather simple approximation allows for a consistent description of the overpotential dependencies on Ag, $\mathrm{Au}-\mathrm{Ag}$ hybrid and $\mathrm{Au}$ electrodes by TR-SERR and TR-SEIRA experiments [40], but further experimental studies and a refinement of the theoretical model are required.

\section{Electron transfer in the high-field regime}

Molecular dynamics simulations of cytochrome $c$ immobilized on electrodes coated with carboxyl-terminated SAMs have identified two main binding domains, differing with respect to the haem plane orientation relative to the surface normal $[42,43]$. The thermodynamically preferred high-affinity binding domain, composed of Lys72, Lys73, Lys79, Lys86, and Lys87, is associated with a distinctly weaker average electronic coupling of the haem with the electrode than the medium-affinity binding domain involving Lys 25 and Lys27. As long as the rotational diffusion of the protein on the SAM surface is fast in comparison with electron tunnelling, electron transfer is expected to occur mainly via the orientation of the highest electron tunnelling probability. This is the case in the low-field regime, as demonstrated by the comparison of the electron transfer and reorientation rate constants [28]. With increasing field strength, protein reorientation is increasingly restricted, due to an electric field-dependent increase in the activation barrier for the transition between the various protein orientations. Thus, electron transfer is modulated by the orientation dynamics of the immobilized protein, as reflected by the viscosity dependence of the experimentally determined overall rate constant. Upon further increasing of the field strength, and thus increasing mobility restrictions of the protein, the contribution of orientational dynamics decreases, and electron transfer will largely occur via all orientations that the protein can adopt upon electrostatic binding. Thus, the heterogeneous electron transfer is a convolution of the orientation-dependent electron tunnelling and the orientational distribution of the immobilized protein, and is thus characterized by an apparent electron transfer rate constant, $k_{\text {app }}$. In addition, the electric field effect on electron tunnelling itself, which is not negligible even in the low-field regime, is expected to play a dominant role at high electric fields, and may account for the decrease in $k_{\text {app }}$ when the SAM thickness is reduced below that of a C5-SAM [40], or when the charge density in the interface is increased [16]. Under these extreme conditions, $k_{\text {app }}$ displays a kinetic isotope effect that is tentatively attributed to the field-dependent reorganization of the hydrogen bond network in the protein-SAM interface [40]. Altogether, the interfacial redox processes in the high-field regime reflect a complex interplay of various (field-dependent) elementary steps that should lead to nonexponential kinetics of the overall electron transfer [44]. Unfortunately, the accuracy, time resolution and dynamic range of TR-SERR and TR-SEIRA spectroscopy are currently not sufficient to disentangle the overall kinetics in this regime, which are therefore approximated by monoexponential behaviour.

\section{Electric field effects on the function of cytochrome $c$}

The electric field-dependent modulation of the electron transfer mechanism and dynamics has been suggested to play a role in the natural redox processes of cytochrome $c$ with the mitochondrial membrane-bound enzyme complexes III and IV [7]. It has been proposed that, in general, these processes take place under lowfield conditions that ensure rapid interprotein electron transfer [16]. However, a transient increase in the transmembrane potential may cause an intermediate transition to the high-field regime, such that the redox reactions of cytochrome $c$, and possibly also intramolecular charge transfer processes in complexes III and IV, are slowed down. Such an increase in the potential may occur if the transmembrane proton gradient produced during the enzymatic process, inter alia, of complex IV is not immediately degraded by ATPase, 
thereby constituting a feedback inhibition to avoid unproductive consumption of molecular oxygen. This hypothesis is difficult to check, although previous studies on complex IV reconstituted in liposomes have shown that both the intramolecular charge transfer processes and the redox reaction with cytochrome $c$ can be significantly retarded upon raising of the transmembrane ion gradient [45-47].

SERR spectroscopic studies on SAM-coated electrodes have demonstrated that very high electric fields induce a conformational transition from the native form (denoted as state B1) to the conformational state (state B2) (Fig. 4) [27]. In this state, the axial Met80 ligand is removed from the haem iron, leading to a coordination equilibrium between a five-coordinated and a six-coordinated species, in which this axial coordination site remains vacant or is occupied by a histidine (His33 or His26) [48]. This structural transition, which is associated with a decrease in the redox potential by $300-400 \mathrm{mV}$, also occurs when cytochrome $c$

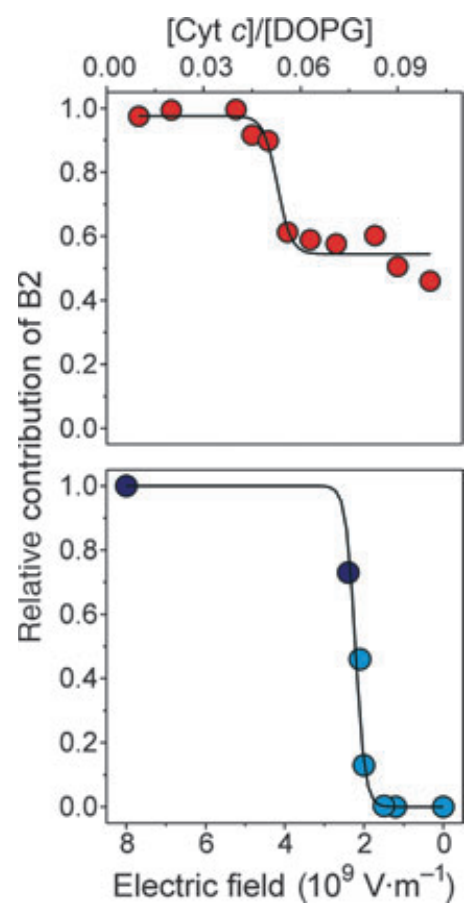

Fig. 4. Relative contributions of the B2 states for ferric cytochrome $c$ (Cyt c) bound to dioleoyl-phosphatidylglycerol (DOPG) vesicles as a function of the protein/lipid ratio, determined by $R R$ spectroscopy (top) [49], and for cytochrome $c$ bound to coated Ag electrodes as a function of the electric field strength, determined by SERR spectroscopy (bottom) [23]. The latter plot includes data from electrodes with carboxyl-terminated SAMs (CX-SAM, with $x=15,10,5,2,1$; light blue), and sulphate and $\mathrm{C}_{11}-\mathrm{PO}_{3}$ coatings (dark blue) [16]. The solid lines are included to guide the eyes. binds to liposomes of negatively charged phospholipids, specifically at low protein/lipid ratios corresponding to high local electric fields (Fig. 4) $[27,48,49]$. It is therefore possible that state B2 may also be formed when cytochrome $c$ binds to the inner mitochondrial membrane, which includes the anionic cardiolipin as the main lipid component. Under physiological conditions, this conformational transition would be associated with a change in protein function, because, owing to the low redox potential, state B2 cannot be reduced by complex III, abolishing cytochrome $c$ 's function as an electron carrier. On the other hand, loss of the Met80 ligand strongly increases the peroxidase activity [50], which may account for the cytochrome $c$-dependent peroxidation of cardiolipin and the resultant increase in the permeability of the inner mitochondrial membrane [9]. This process is considered to be functional for the release of cytochrome $c$ to the cytosol, where the protein may be involved in caspase-dependent apoptotic pathways [8]. Even though other factors may contribute to the transformation of cytochrome $c$ from an electron carrier to a peroxidase, local electric fields are likely to promote this transition. Indeed, computational studies have shown that, although the intrinsic stability of the $\mathrm{Fe}-\mathrm{S}(\mathrm{Met})$ bond is not significantly affected by biologically meaningful electric fields [51], homogeneous fields of $\sim 25 \mathrm{mV} \cdot \AA^{-1}$ are able to perturb flexible segments of the protein, favouring the transition [43].

\section{Acknowledgements}

This work was supported by the Cluster of Excellence 'UniCat', funded by the DFG (P. Hildebrandt), ANPCyT (PICT2006-459), UBA (UBACyT 200200 90100094) (D. H. Murgida), and the National Science foundation of China (Nos. 20905021) (J.-J. Feng).

\section{References}

1 Prudencio M \& Ubbink M (2004) Transient complexes of redox proteins: structural and dynamic details from NMR studies. J Mol Recognit 17, 524-539.

2 Bashir Q, Scanu S \& Ubbink M (2011) Dynamics in electron transfer protein complexes. FEBS J 278, 13911400 .

3 Martínez-Fábregas L, Rubio S, Díaz-Quintana A, Díaz-Moreno I \& De la Rosa M (2011) Proteomic tools for the analysis of transient interactions between metalloproteins. FEBS J 278, 1401-1410.

4 Murgida DH \& Hildebrandt P (2008) Disentangling interfacial redox processes of proteins by SERR spectroscopy. Chem Soc Rev 37, 937-945. 
5 Clarke RJ (2001) The dipole potential of phospholipid membranes and methods for its detection. Adv Colloid Interface Sci 89, 263-281.

6 Neumann E (1986) Chemical electric effects in biological macromeolecules. Prog Biophys Mol Biol 47, 197-231.

7 Scott RA \& Mauk AG, eds (1995) Cytochrome c-A Multidisciplinary Approach. University Science Books, Sausalito.

8 Jiang X \& Wang X (2004) Cytochrome $c$-mediated apoptosis. Annu Rev Biochem 73, 87-106.

9 Kagan VE, Tyurin VA, Jiang J, Tyurina YY, Ritov VB, Amoscato AA, Osipov AN, Belikova NA, Kapralov AA, Kini V et al. (2005) Cytochrome $c$ acts as a cardiolipin oxygenase required for release of proapoptotic factors. Nat Chem Biol 1, 223-232.

10 Siebert F \& Hildebrandt P (2007) Vibrational Spectroscopy in Life Science. Wiley-VCH, Weinheim.

11 Murgida D \& Hildebrandt P (2001) Active site structure and dynamics of immobilized cytochrome $c$ on selfassembled monolayers - a time-resolved surface enhanced resonance spectroscopic study. Angew Chem Int Ed 40, 728-731.

12 Ataka K \& Heberle J (2003) Electrochemically induced surface enhanced infrared difference absorption (SEIDA) spectroscopy of a protein monolayer. $J \mathrm{Am}$ Chem Soc 125, 4986-4987.

13 Feng JJ, Gernert U, Sezer M, Kuhlmann U, Murgida DH, David C, Richter M, Knorr A, Hildebrandt P \& Weidinger I (2009) A novel Au-Ag hybrid device for surface enhanced (resonance) Raman spectroscopy. Nano Lett 9, 298-303.

14 Sezer M, Feng JJ, Ly KH, Shen Y, Nakanishi T, Kuhlmann U, Möhwald H, Hildebrandt P \& Weidinger I (2010) Multi-layer electron transfer across nanostructured Ag-SAM-Au-SAM junctions probed by surface enhanced Raman spectroscopy. Phys Chem Chem Phys 12, 9822-9829.

15 Feng JJ, Gernert U, Hildebrandt P \& Weidinger IM (2010) Novel nano-sandwiched Ag-silica-Au supports with high SER-activity via long range plasmon coupling. Adv Funct Mat 20, 1954-1961.

16 Murgida DH \& Hildebrandt P (2005) Redox and redox-coupled processes of heme proteins and enzymes at electrochemical interfaces. Phys Chem Chem Phys 7, 3773-3784.

17 Love JC, Estroff LA, Kriebel JK, Nuzzo RG \& Whitesides GM (2005) Self-assembled monolayers of thiolates on metals as a form of nanotechnology. Chem Rev 105, 1103-1169.

18 Suydam IT, Snow CD, Pande VS \& Boxer SG (2006) Electric fields at the active site of an enzyme: direct comparison of experiment with theory. Science $\mathbf{3 1 3}$, 200-204.

19 Fafarman AT, Webb LJ, Chuang JI \& Boxer SG (2006) Site-specific conversion of cysteine thiols into thiocya- nate creates an IR probe for electric fields in proteins. J Am Chem Soc 128, 13356-13357.

20 Suydam IT \& Boxer SG (2003) Vibrational Stark effects calibrate the sensitivity of vibrational probes for electric fields in proteins. Biochemistry 42, 12050-12055.

21 Farfarman AT, Sigala PA, Herschlag D \& Boxer SG (2010) Decomposition of vibrational shifts of nitriles into electrostatic and hydrogen bonding effects. $J$ Am Chem Soc 132, 12811-12813.

22 Oklejas V \& Harris JM (2003) In-situ investigation of binary-component self-assembled monolayers: a SERSbased spectroelectrochemical study of the effects of monolayer composition on interfacial structure. Langmuir 19, 5794-5801.

23 Murgida DH \& Hildebrandt P (2001) The heterogeneous electron transfer of cytochrome $c$ adsorbed on coated silver electrodes. Electric field effects on structure and redox potential. $J$ Phys Chem B $\mathbf{1 0 5}$, 1578-1586.

24 Armstrong FA (2005) Recent developments in dynamic electrochemical studies of adsorbed enzymes and their active sites. Curr Opin Chem Biol 9, 110-117.

25 Murgida DH \& Hildebrandt P (2001) Proton coupled electron transfer in cytochrome c. J Am Chem Soc 123, 4062-4068.

26 Wisitruangsakul N, Zebger I, Ly KH, Murgida DH, Egkasit S \& Hildebrandt P (2008) Redox-linked protein dynamics probed by time-resolved surface enhanced infrared absorption spectroscopy. Phys Chem Chem Phys 10, 5276-5286.

27 Wackerbarth H \& Hildebrandt P (2003) Redox and conformational equilibria and dynamics of cytochrome $c$ at high electric fields. ChemPhysChem 4, 714-724.

28 Kranich A, Ly HK, Hildebrandt P \& Murgida DH (2008) Direct observation of the gating step in protein electron transfer: electric field controlled protein dynamics. J Am Chem Soc 130, 9844-9848.

29 Feng ZQ, Imabayashi S, Kakiuchi T \& Niki K (1997) Long-range electron-transfer reaction rates to cytochrome $c$ across long- and short-chain alkanethiol self-assembled monolayers: electroreflectance studies. J Chem Soc Faraday Trans 93, 1367-1370.

30 Avila A, Gregory BW, Niki K \& Cotton TM (2000) An electrochemical approach to investigate gated electron transfer using a physiological model system: cytochrome $c$ immobilized on carboxylic acid-terminated alkanethiol self-assembled monolayers on gold electrodes. J Phys Chem B 104, 2759-2766.

31 Niki K, Hardy WR, Hill MG, Li H, Sprinkle JR, Margoliash E, Fujita K, Tanimura R, Nakamura N, Ohno H et al. (2003) Coupling to lysine-13 promotes electron tunneling through carboxylate-terminated alkanethiol self-assembled monolayers to cytochrome $c$. J Phys Chem B 107, 9947-9949. 
32 Wei JJ, Liu HY, Khoshtariya DE, Yamamoto H, Dick A \& Waldeck DH (2002) Electron-transfer dynamics of cytochrome $c$ : a change in the reaction mechanism with distance. Angew Chem Int Ed 41, 4700-4703.

33 Murgida DH, Hildebrandt P, Wei J, He YF, Liu HY \& Waldeck DH (2004) SERR and electrochemical study of cytochrome $c$ bound on electrodes through coordination with pyridinyl-terminated SAMs. J Phys Chem B 108, 2261-2269.

34 Wei JJ, Liu HY, Niki K, Margoliash E \& Waldeck DH (2004) Probing electron tunneling pathways: electrochemical study of rat heart cytochrome $c$ and its mutant on pyridine-terminated SAMs. J Phys Chem B 108, 16912-16917.

35 Yue HJ \& Waldeck DH (2005) Understanding interfacial electron transfer to monolayer protein assemblies. Curr Opin Solid State Mater Sci 9, 28-36.

36 Khoshtariya DE, Wei JJ, Liu HY, Yue HJ \& Waldeck DH (2003) Charge-transfer mechanism for cytochrome $c$ adsorbed on nanometer thick films. Distinguishing frictional control from conformational gating. J Am Chem Soc 125, 7704-7714.

37 Yue HJ, Khoshtariya D, Waldeck DH, Grochol J, Hildebrandt P \& Murgida DH (2006) On the electron transfer mechanism between cytochrome $c$ and metal electrodes. Evidence for dynamic control at short distances. J Phys Chem B 110, 19906-19913.

38 Murgida D \& Hildebrandt P (2002) Electrostatic-field dependent activation energies control biological electron transfer. J Phys Chem B 106, 12814-12819.

39 Cheng J, Terrettaz S, Blankman JI, Muller CJ, Dangi B \& Guiles RD (1997) Electrochemical comparison of heme proteins by insulated electrode voltammetry. Isr $J$ Chem 37, 259-266.

40 Ly KH, Wisitruangsakul N, Sezer M, Feng JJ, Kranich A, Weidinger I, Zebger I, Murgida DH \& Hildebrandt P (2010) Electric field effects on the interfacial electron transfer and protein dynamics of cytochrome c. J Electroanal Chem, in press, doi:10.1016/j.jelechem.2010.12. 020 .

41 Lockhart DJ, Kirmaier C, Holten D \& Boxer SG (1990) Electric field effects on the initial electron-transfer kinetics in bacterial photosynthetic reaction centers. J Phys Chem 94, 6987-6995.
42 Paggi D, Martín DF, Kranich A, Hildebrandt P, Martí M \& Murgida DH (2009) Computer simulation and SERR detection of cytochrome $c$ dynamics at SAM-coated electrodes. Electrochim Acta 54, 4963-4970.

43 De Biase PM, Paggi DA, Doctorovich F, Hildebrandt P, Estrin DA, Murgida DH \& Marti MA (2009) Molecular basis for the electric field modulation of cytochrome $c$ structure and function. J Am Chem Soc 131, 16248-16256.

44 Georg S, Kabuss J, Weidinger IM, Murgida DH, Hildebrandt P, Knorr A \& Richter M (2010) On the distance dependent electron transfer rate of immobilised redox proteins - a statistical physics approach. Phys Rev E 81, 046101(1-10).

45 Moroney PM, Scholes TA \& Hinkle PC (1984) Effect of membrane-potential and $\mathrm{pH}$ gradient on electrontransfer in cytochrome-oxidase. Biochemistry 23, 4991-4997.

46 Gregory LS \& Ferguson-Miller S (1989) Independent control of respiration in cytochrome-c oxidase vesicles by $\mathrm{pH}$ and electrical gradients. Biochemistry 28, 26552662.

47 Sarti P, Antonini G, Malatesta F \& Brunori M (1992) Respiratory control in cytochrome-oxidase vesicles is correlated with the rate of internal electron-transfer. Biochem J 284, 123-127.

48 Oellerich S, Wackerbarth H \& Hildebrandt P (2002) Spectroscopic characterization of non-native states of cytochrome $c$. J Phys Chem B 106, 6566-6580.

49 Oellerich S, Lecomte S, Paternostre M, Heimburg T \& Hildebrandt P (2004) Peripheral and integral binding of cytochrome $c$ to phospholipid vesicles. J Phys Chem B 108, 3871-3878.

50 Basova LV, Kurnikov IV, Wang L, Ritov VB, Belikova NA, Vlasova II, Pacheco AA, Winnica DE, Peterson J, Bayir H et al. (2007) Cardiolipin switch in mitochondria: shutting off the reduction of cytochrome $c$ and turning on the peroxidase activity. Biochemistry 2007, 46.

51 De Biase PM, Doctorovich F, Murgida DH \& Estrin DA (2007) Electric field effects on the reactivity of heme model systems. Chem Phys Lett 434, 121-126. 